Best Oral (BO5)

ERAS

https://doi.org/10.3802/jgo.2021.32.S1.BO5

\title{
Effectiveness of Enhanced Recovery After Surgery protocol in open gynecologic oncology surgery: a randomized controlled trial
}

\author{
Phasawee Chiewhatpong, ${ }^{1}$ Kittipat Charoenkwan, ${ }^{1}$ Kannika Smithiseth, ${ }^{2}$ Warangkana Lapisatepun, ${ }^{2}$ \\ Panuwat Lapisatepun, ${ }^{2}$ Mattabhorn Phornphutkul,, ${ }^{3}$ Tanarat Muangmool,' Chalong Cheewakriangkrai, \\ Prapaporn Suprasert' Jatupol Srisomboon'
}

\begin{abstract}
'Department of Obstetrics and Gynecology, Faculty of Medicine, Chiang Mai University, Chiang Mai, Thailand (charoenkwan@live.com) ${ }^{2}$ Department of Anesthesiology, Faculty of Medicine, Chiang Mai University, Chiang Mai, Thailand

${ }^{3}$ Department of Internal Medicine, Faculty of Medicine, Chiang Mai University, Chiang Mai, Thailand
\end{abstract}

Objective: Standard guidelines strongly recommends Enhanced Recovery After Surgery (ERAS) protocol implementation into each institution since studies have shown its effectiveness in reducing length of hospital stay (LOS) and rate of postoperative complications. In order to extend the benefits of ERAS globally especially to the low- and middle-income countries, where healthcare resources are limited, it is necessary to tailor the ERAS protocol to make it practical and cost-effective for each region. To examine the effectiveness and feasibility of fully applying the recommended ERAS protocol in comparison to our usual care in women diagnosed with gynecologic malignancy undergoing elective laparotomy.

Methods: This is a randomized controlled trial in women with gynecologic cancers (cervix, endometrium, and ovary), age 18-75 years, undergoing elective laparotomy at our institution. The participants were randomly assigned into 2 groups: intervention (ERAS protocol) and control (usual care). For the intervention group, each woman was brought through the pre-specified protocol step by step starting from preoperative counseling and preparation to intraoperative and postoperative management according to the recommended ERAS pathway. For the control group, each woman was cared for using routine standard care. The primary outcomes were LOS and postoperative pain. The secondary outcomes were narcotic use, mobility, return of gastrointestinal function, postoperative complications, and quality of life. Furthermore, the compliance with each ERAS component was examined. Results: From June 2020 to May 2021, 93 women (46 in the intervention group and 47 in the control group) participated in the allocated study groups through the study completion and were included in the final analysis. The intervention group demonstrated shorter hospital stay by approximately 20 hours ( 47.48 hours vs. 67.17 hours, $\mathrm{p}=0.02$ ) with lower postoperative pain score at postoperative day 0 ( 1.58 vs. 4.00, $\mathrm{p}<0.01)$ and day 1 (1.00 vs. 2.67, $\mathrm{p}<0.01)$ while having decreased opioid consumption $(\mathrm{p}<0.01)$. Also, the intervention group had faster postoperative recovery of gastrointestinal function represented by significantly shorter interval from surgery to first flatus ( $\mathrm{p}<0.01)$, first defecation $(\mathrm{p}=0.02)$, and soft diet feeding $(\mathrm{p}<0.01)$. Postoperative 6 -minute walking distance, postoperative complications, and quality of life were comparable between the study groups. Good compliance to most of the ERAS pathway domains was obtained. Still, 34.8\% of participants in the ERAS group had bowel preparation and $10.9 \%$ had preanesthetic sedatives.

Conclusion: The ERAS protocol appeared feasible in our population with demonstrable benefits on shortening hospital stay, reducing pain, and promoting bowel function recovery without increasing complications.

Trial Registration: ClinicalTrials.gov Identifier: NCT04201626 